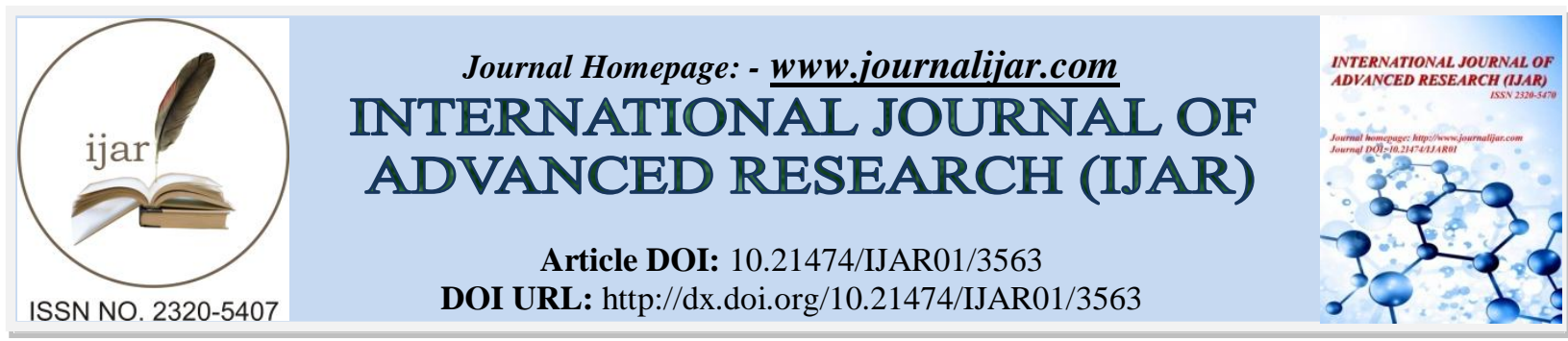

RESEARCH ARTICLE

\title{
EVALUATION OF LEFT VENTRICULAR DIASTOLIC FUNCTION IN PREGNANCY INDUCED HYPERTENSION AND ITS COMPARISON WITH NORMOTENSIVE PREGNANT ASIAN INDIAN FEMALES.
}

\section{Dr. Suresh Patted ${ }^{1}$, Dr. S. C. Porwal ${ }^{2}$, Dr. Prabhu C. Halkati ${ }^{3}$, Dr. Sameer Ambar ${ }^{2}$, Dr. Prasad M.R. ${ }^{2}$,} Dr. Vijay Metgudmath ${ }^{2}$, Dr. Vishwanath $\mathrm{H}^{4}$, Dr. Vaibhav Patil ${ }^{2}$ and Dr. Ankur Batra ${ }^{5}$

1. Head of Department, Department of Cardiology, JN Medical College, KLE University, Belagavi.

2. Associate Professor, Department of Cardiology, JN Medical College, KLE University, Belagavi.

3. Professor, Department of Cardiology, JN Medical College, KLE University, Belagavi.

4. Senior Resident, Department of Cardiology, JN Medical College, KLE University, Belagavi.

5. Junior Resident, Department of Cardiology, JN Medical College, KLE University, Belagavi.

\section{Manuscript Info}

\section{Manuscript History}

Received: 03 January 2017

Final Accepted: 02 February 2017

Published: March 2017

Key words:-

Diastolic Dysfunction, Pregnancy

Induced Hypertension, 2D

Echocardiography

\section{Abstract}

Introduction: Pregnancy related increased preload alters the diastolic properties of the left ventricle which have negative effects on maternal health. Scarcity of data exist on the diastolic properties of left ventricle in pregnancy induced hypertension.

Objectives: To evaluate the Left ventricular diastolic dysfunction in pregnancy induced hypertension and its comparison with normotensive pregnant females using standard 2D echocardiography and Doppler studies.

Methods: This is a prospective study done in Department of Cardiology, KLES Dr Prabhakar Kore Hospital,Belagavi,India.

The study population consisted of 100 hypertensive $(\mathrm{H})$ and 100 normotensive(N) pregnant females which were evaluated by $2 \mathrm{D}$ Echocardiography and Doppler parameters for diastolic dysfunction of Left ventricle.

Diastolic parameters studied were mitral valve $\mathrm{E}$ wave, A wave, E/A ratio, isovolumetric relaxation time (IVRT), E deceleration time, Deceleration slope, pulmonary vein systolic (PVs), diastolic (PVd) and its ratio, $\mathrm{E}$ wave velocity time integral, $\mathrm{A}$ wave velocity time integral and tissue Doppler study of the mitral valve annulus.

Results :Majority of the women in group N(94\%) had normal diastolic function that is grade 0,compared to group $\mathrm{H}(56 \%)$.

This difference was statistically significant $(\mathrm{p}<0.001)$.

Most significantly associated characteristics with diastolic dysfunction included MV: E/E' $(p=0.004)$, IVRT $(p<0.001)$, Deceleration Slope $(\mathrm{p}<0.001), \mathrm{PVa}(<0.001)$ and $\mathrm{PVs}(0.015)$.

Mean grade of diastolic dysfunction in hypertensives was significantly high compared to normotensives $(\mathrm{p}<0.001)$.

Conclusions: This study concludes that there is significant incidence of diastolic dysfunction in pregnancy induced hypertension as compared to normotensive Asian Indian females. 
LV inflow E/E', Deceleration slope, IVRT and Pulmonary vein flow velocities are most significant echocardiographic characteristic associated with diastolic dysfunction in pregnancy induced hypertension.

Copy Right, IJAR, 2017,. All rights reserved.

\section{Introduction:-}

Pregnancy introduces a plethora of hemodynamic challenges to the maternal cardiovascular system which is further intensified in pregnancy induced hypertension.Pregnancy induced hypertension includes gestational hypertension, pre-eclampsia, and eclampsia. It is associated with increased maternal morbidity and mortality. The incidence of hypertensive disorders ranges from $2-8 \%$ of all pregnancies and contributes to $9 \%$ of maternal mortality in Asia and $12 \%$ in India. ${ }^{1,2}$

Pre-eclampsia involves multiple systems in mother and can lead to fetal mortality and morbidity secondary to uteroplacental insufficiency. $^{3}$

Gestational hypertension is diagnosed when the BP exceeds 140/90 in the absence of proteinuria or pathologic edema in a previously normotensive female at or after 20 weeks of gestation.Pre-eclampsia is considered severe when proteinuria exceeds $4 \mathrm{gm} / 24$ hours, blood pressure is or more than 160/110 and/or severe headache, visual disturbances or epigastric pain is noted. Eclampsia is the development of generalized seizures in a patient of preeclampsia. ${ }^{1}$ The incidence in primigravidae is about $10 \%$ and in multigravidae about $5 \% .^{4}$

Pregnancy induced hypertension increases cardiopulmonary morbidity and has a higher prevalence of myocardial contraction band necrosis at autopsy suggesting that pre-eclampsia may be associated with potentially significant myocardial damage. ${ }^{5}$

During normal hemodynamic adaptations there is some degree of diastolic dysfunction even in normotensive pregnancy which gets further exaggerated in pre-eclampsia leading to increased cardiovascular morbidity and mortality. ${ }^{6,7,8}$ Normal diastolic function allows adequate filling of ventricles during rest and exercise without an abnormal increase in filling pressures. Initial diastolic dysfunction is impaired relaxation and with further progression of disease, there is mild to moderate increase in left atrial pressure and the mitral inflow velocity pattern appears similar to a normal filling pattern (pseudo normalization). However with further progression of the disease, the pattern becomes restrictive and finally irreversible. Doppler echocardiography is an excellent and routinely used non-invasive tool to assess the diastolic function of the heart.

This study is designed to find out the prevalence of diastolic dysfunction in pregnancy induced hypertension as compared to normotensive pregnant women by 2D Echocardiography and Doppler indices.

\section{Methods:-}

A case control study was performed between January 2015 and January 2016 in the Department of Cardiology, KLEs Dr Prabhakar Kore Hospital and Medical Research Centre, Belagavi. 100 singleton pregnant women with gestational hypertension, were enrolled for the study during the third trimester of pregnancy and were matched with 100 controls.Gestational hypertension was diagnosed according to Davey and MacGillivray. ${ }^{9}$ For each patient with gestational hypertension, a normotensive woman was matched. Approval of the University Ethics Committee was obtained, and written informed consent was collected from all patients.

Exclusion criteria included the following: undetermined gestational age, history of heart disease, undetermined development of hypertension, previously on antihypertensive treatment, and comorbidities namely diabetes, renal failure, anaemia and not in sinus rhythm. Blood pressure was measured from right arm in supine position.

\section{Echocardiographic evaluation:-}

2D Echo and Doppler data were obtained in patients in left lateral position with a 2.0 to $80 \mathrm{MHz}$ transducer interfaced with a commercially available echocardiographic machine (Phillips IE33).Hypertensive patients were studied prior to initiation of treatment.Severity and grading of diastolic dysfunction was done by calculating Doppler 
indices namely transmitral and pulmonary venous flow patterns recorded in the apical 4-chamber view.

Mitral flow velocities were detected by placing the sample volume between the tips of the mitral leaflets.

The following variables were measured:

1. Peak flow velocity in early diastole (E wave) and during atrial contraction (A wave);

2. Peak E/A ratio;

3. E- and A-wave time velocity integrals (E-VTI, A-VTI);

4. Deceleration time of the E wave (DT); and

5. Left ventricular isovolumetric relaxation time (IVRT).

6. Pulmonary venous flow velocities namely during ventricular systole (PVs) and its time-velocity integral (PVs$\mathrm{VTI})$; peak pulmonary venous flow velocity during ventricular diastole $(\mathrm{PVd})$ and its time-velocity integral (PVd-VTI);peak pulmonary venous flow velocity at atrial contraction (PVa) and its time-velocity integral (PVaVTI); and the duration of the PVa (dPVa).

\section{Statistical Analysis:-}

Data was analysed using SPSS statistical software version 20.0 The categorical data was expressed as rates, ratios and proportions and comparison was done using either Chi-square test or Fisher's exact test. The continuous data was expressed as mean \pm standard deviation (SD) and comparison was done using independent sample ' $\mathrm{t}$ ' test. A probability value ('p' value) of less than or equal to 0.05 was considered as statistically significant. Observer bias was avoided by taking in measurements by 2 different observers from random selected patient data recordings.

\section{Results:-}

The present study included a total of 200 pregnant women that is, 100 women with hypertension (Group H) and 100 women with normal pregnancy (Group N). The comparison of patient and echocardiographic characteristics of the study population is tabulated in Table 1.

Table 1:- Patient and Echocardiographic characteristics of the study population

\begin{tabular}{|l|l|l|l|l|l|}
\hline \multirow{2}{*}{ Characteristics } & \multicolumn{2}{l|}{ Group N (n=100) } & \multicolumn{2}{l|}{ Group (n=100) } & \multirow{2}{*}{ p value } \\
\cline { 2 - 5 } & Mean & SD & Mean & SD & \\
\hline Age & 23.06 & 2.92 & 23.48 & 2.38 & $\mathbf{0 . 0 3 2}$ \\
\hline Gestational age (weeks) & 35.80 & 0.57 & 35.78 & 0.51 & $\mathbf{0 . 0 4 4}$ \\
\hline MV:E & 5.60 & 1.80 & 8.11 & 9.05 & 0.060 \\
\hline MV: A & 57.70 & 25.04 & 56.75 & 27.93 & 0.858 \\
\hline MV: E/A & 1.25 & 1.48 & 1.39 & 1.58 & 0.643 \\
\hline MV:E' & 7.23 & 3.01 & 8.53 & 4.55 & 0.096 \\
\hline MV:A' & 9.99 & 5.53 & 9.36 & 3.27 & 0.491 \\
\hline MV:E/E' & 33.41 & 54.32 & 10.02 & 25.29 & $\mathbf{0 . 0 0 4}$ \\
\hline IVRT & 144.02 & 20.15 & 119.96 & 31.91 & $<\mathbf{0 . 0 0 1}$ \\
\hline DT & 130.88 & 29.04 & 173.24 & 152.68 & 0.059 \\
\hline DS & 294.73 & 166.10 & 487.92 & 208.62 & $<\mathbf{0 . 0 0 1}$ \\
\hline PVs & 43.30 & 13.38 & 50.24 & 14.52 & $\mathbf{0 . 0 1 5}$ \\
\hline PVd & 43.98 & 12.45 & 46.64 & 13.85 & 0.315 \\
\hline PVa & 31.58 & 8.00 & 40.52 & 11.31 & $<\mathbf{0 . 0 0 1}$ \\
\hline PVs/PVd & 1.12 & 0.43 & 1.16 & 0.38 & 0.591 \\
\hline Systolic Blood Pressure & 121.88 & 5.51 & 140.16 & 12.47 & $<\mathbf{0 . 0 0 1}$ \\
\hline Diastole Blood Pressure & 79.70 & 9.40 & 86.00 & 7.44 & $<\mathbf{0 . 0 0 1}$ \\
\hline & & & & & \\
\hline It wannnnn
\end{tabular}

It was observed that, the mean maternal age and gestational age were comparable in both the groups (p $<0.05)$. Systolic $(\mathrm{p}<0.001)$ and diastolic $(\mathrm{p}<0.001)$ blood pressure was significantly high in group H compared to group N. Amongst all the Doppler characteristics MV: E/E' $(p=0.004)$, IVRT $(p<0.001)$, DS $(p<0.001)$, PVs $(p=$ $0.015)$ and $\mathrm{PVa}(\mathrm{p}<0.001)$ were significantly higher in Group H when compared to Group N.

On the basis of the above mentioned Doppler characteristics the grade of diastolic dysfunction was evaluated.In the present study majority of the women in group $\mathrm{N}$ had normal diastolic function that is grade 0 in $94 \%$ compared to $56 \%$ in group $\mathrm{H}$. This difference was statistically highly significant $(\mathrm{p}<0.001)$.(Table 2$)$ 
Table 2:- Distribution of the study population according to Grade of diastolic dysfunction

\begin{tabular}{|c|c|c|c|c|c|c|}
\hline \multirow{2}{*}{$\begin{array}{l}\text { Grade of Diastolic } \\
\text { dysfunction }\end{array}$} & \multicolumn{2}{|c|}{ Group N (n=100) } & \multicolumn{2}{|c|}{ Group H $(n=100)$} & \multirow[t]{2}{*}{$x^{2}$} & \multirow[t]{2}{*}{ p value } \\
\hline & No. & $\%$ & No. & $\%$ & & \\
\hline 0 & 94 & 94.00 & 56 & 56.00 & 19.379 & $<0.001$ \\
\hline 1 & 6 & 6.00 & 40 & 40.00 & & \\
\hline 2 & 0 & 0.00 & 4 & 4.00 & & \\
\hline Total & 100 & 100.00 & 100 & 100.00 & & \\
\hline
\end{tabular}

\section{Discussion:-}

Pregnancy induced hypertension is characterized by progressive hypertension during gestation and may be associated with pathological edema and proteinuria. It complicates $2-8 \%$ of pregnancies. ${ }^{1,2}$

Pregnancy induced hypertension has a placental pathogenesis with acute onset of cardiovascular manifestations secondary to generalized endothelial dysfunction and vasospasm resulting in hypertension and multi-organ hypoperfusion. The major scientific societies provide different criteria for the diagnosis of pregnancy induced hypertension. Common to all is new-onset hypertension ( $\geq 140 \mathrm{~mm} \mathrm{Hg}$ systolic blood pressure [SBP] or $\geq 90 \mathrm{~mm} \mathrm{Hg}$ diastolic blood pressure [DBP]) arising after 20 weeks of gestation and complete resolution within 12 weeks postpartum. The term pre-eclampsia and eclampsia indicates presence of proteinuria and generalized tonic clonic seizures associated with hypertension respectively. It is one of the leading causes of maternal morbidity and mortality worldwide, and delay in the treatment of severe hypertension and diagnosis of pre-eclampsia complications contribute significantly to maternal mortality. ${ }^{5}$ Multiple exceptional and exclusive changes in cardiac structure and function have been described in pre-eclampsia, suggesting that these women display abnormal cardiac adaptation to pregnancy and hence towards long-term postpartum cardiovascular disease (CVD). The development of pre-eclampsia and eclampsia is now considered a risk factor for long-term CVD. ${ }^{5}$

The data on changes in diastolic function in pregnancy induced hypertension is limited and studies have reported varying levels of diastolic function across various stages of gestation. ${ }^{10}$

Therefore,this study was undertaken to assess changes in diastolic function in pregnancy induced hypertension and compared to normal pregnant women by 2D echocardiography and Doppler evaluation.

This was a one year prospective study, conducted in the Department of Cardiology with 200 pregnant women that is, 100 women with pregnancy induced hypertension (Group H) and 100 women with normal pregnancy (Group N). All the women underwent 2D echo for the evaluation of diastolic function.

In this study majority of subjects were between 21 to 25 year of age i.e. $66 \%$ in Group $\mathrm{H}$ and $62 \%$ in Group N. Also the mean age in group $\mathrm{H}$ was $23.48 \pm 2.38$ years and in group $\mathrm{N}$ it was $23.06 \pm 2.92$ years $(\mathrm{p}=0.032)$ suggesting that the age distribution of the study population in group $\mathrm{H}$ and group $\mathrm{N}$ was comparable.

In this study,92\% women in Group $\mathrm{H}$ and $78 \%$ in Group $\mathrm{N}$ had gestational age of 36 weeks. The mean gestational age in group $\mathrm{H}$ was $35.78 \pm 0.51$ years and in group $\mathrm{N}$ it was $35.80 \pm 0.57$ years $(\mathrm{p}=0.044)$. Hence the gestational age in group $\mathrm{H}$ and group $\mathrm{N}$ was comparable. These findings rule out the possible bias in study results.

In the present study $2 \mathrm{D}$ echocardiography revealed majority of the women in group $\mathrm{N}$ with normal diastolic function that is grade 0 in $94 \%$ while in group $\mathrm{H} 56 \%$ of the women have normal diastolic function. This difference was statistically highly significant $(\mathrm{p}<0.001)$.

Furthermore, Grade 1 diastolic dysfunction was present in significantly higher number of women with pre-eclampsia compared to women with normal pregnancy ( $40 \%$ vs $6 \%$ ) and Grade II diastolic dysfunction was noted in $4 \%$ of the women with pre-eclampsia while none of the normal women was found to have Grade II diastolic dysfunction.

Hence these findings suggest that, pregnant women with pre-eclampsia are likely to have diastolic dysfunction as assessed by 2D Echocardiography.

In this study Doppler parameters including MV: E/E' $(p=0.004)$, IVRT $(p<0.001)$, DS $(p<0.001)$, PVs $(p=$ $0.015)$ and $\mathrm{PVa}(\mathrm{p}<0.001)$ were significantly high in group $\mathrm{H}$ compared to group $\mathrm{N}$. 
However MV: E, MV: A, MV: E/A, MV: E', MV: A', DTI, PVd and PVs/PVd were found to be comparable but statistically insignificant $(\mathrm{p}>0.05)$.

Hence these findings suggest that, pregnant women with hypertension are likely to have diastolic dysfunction in terms of raised MV: E/E', IVRT, DS, PVs and PVa.

These findings were comparable to other studies.

In a study by Solanki R. et al $^{10}$ authors evaluated maternal cardiovascular function using echocardiography in normal and pre-eclamptic women in the third trimester of pregnancy on 20 subjects with pre-eclampsia and 20 normotensive controls with 34 weeks gestation and singleton pregnancy. Study revealed that increased afterload in pregnancy induced hypertension is linked with reduced emptying of left ventricle. Increased E wave velocity in hypertensive patients suggest elevated trans mitral gradient during early diastole reflecting reduced LV compliance. The higher peak A wave velocity suggests more important role of atrial systole in filling of the hypertrophied ventricle in these women. The prolonged IVRT in pre-eclamptic subjects suggests that longer time is required for left ventricular pressure to fall below atrial pressure. The prolongation of E wave deceleration time in pre-eclamptic subjects is consistent with increased passive filling of left ventricle during early diastole.Mean cardiac output $(66.85 \pm 4.56 \mathrm{ml} / \mathrm{min}$ vs. $56.1 \pm 1.77 \mathrm{ml} / \mathrm{min})$, mean LV diastolic mass $(66.85 \pm 4.56 \mathrm{ml} / \mathrm{min}$ vs. $56.1 \pm 1.77 \mathrm{ml} / \mathrm{min})$ and total vascular resistance $(1396.85 \pm 150.2 \mathrm{vs} .1204 .5 \pm$ 71.182 dyne, s cm5) was higher in the women with pre eclampsia indicating reduced LV compliance and diastolic dysfunction. This study hence concluded that there are significant structural and dynamic changes in women with preeclampsia particularly reduced LV compliance and diastolic dysfunction. Blood pressure monitoring alone is insufficient in identifying and managing the risk of cardiovascular complications in such patients.Including echocardiographic assessment in the management protocol could help identify women who are at higher risk of developing complications.

Melchiorre K, et al. ${ }^{8}$ conducted a prospective case control study comparing the diastolic function of 50 patients with preeclampsia and 50 normal pregnancies. It was undertaken to evaluate cardiac function and LV remodeling occurring at term in patients with pre eclampsia. Global diastolic dysfunction was seen in $40 \%$ of pre eclampsia cases as compared to $14 \%$ in normotensive pregnancies $(\mathrm{p}=0.007)$. Altered indices of LV mass indicate Left ventricular adaptation to maintain myocardial contractility against increased afterload in pre-eclampsia patients. As diastolic dysfunction precedes systolic dysfunction in other causes, its development is of prognostic value in patients with pre-eclampsia. Hence this reiterates the importance of early detection of diastolic dysfunction in women with pre- eclampsia.

\section{Conclusion:-}

Echocardiography can be used as a useful screening tool to identify diastolic dysfunction in patients with pregnancy induced hypertension. The echocardiographic characteristics including Mitral Valve: E/E', IVRT, DS,PVs and PVa were significantly higher in pregnancy induced hypertension when compared to normotensive pregnant females.

\section{References:-}

1. Cunningham FG, Hauth JC, Leveno KJ, Gilstrap L, Bloom SL, Wenstrom KD. William's obstetrics 22nd ed. New York. McGraw Hill publishers; 2005.

2. Khan KS et al, WHO analysis of causes of maternal death: A systematic review. Lancet 2006; 367:1066-74.

3. Sibai, BM. Diagnoses, prevention and management of eclampsia. Obstet Gynecol. 2005; 105: 402-410

4. Chaim SRP, Oliveira SMJ Vasconcellos de, Kimura AF. Pregnancy-induced hypertension and the neonatal outcome. ActaPaulista de Enfermagem 2008;21(1):53-8.

5. Melchiorre K, Sharma R, Basky T. Cardiovascular Implications in Preeclampsia - An Overview.Circulation.2014;130: 703-14

6. Andres M, Carlos J, Antonieta,H Karolina, A, Dale, BWK Vanghn et al. Left Ventricular Diastolic Function in normal human pregnancy.Circulation. 1999;99:511-517

7. Valensise H, Novelli GP, Vasapollo B, Di Ruzza G, Romanini ME, Marchei M, et al. Maternal diastolic dysfunction and left ventricular geometry in gestational hypertension. Hypertension 2001;37:1209-15

8. Melchiorre K, Sutherland GR, Liberati M, Thilaganathan B. Preeclampsia is associated with persistent postpartum cardiovascular impairment. Hypertension. 2011;58(4):709-715

9. Rath W, Fischer T. The Diagnosis and Treatment of Hypertensive Disorders of Pregnancy: New Findings for Antenatal and Inpatient Care. Deutsches Ärzteblatt International. 2009;106(45):733-738.

10. Solanki R, Maitra N. Echocardiographic assessment of cardiovascular

11. hemodynamics in pre-eclampsia. J ObstetGynecol India. 2011;61(5):519-22. 